\title{
REZAGOS DE LA INFLACIÓN EN LA ECONOMÍA VENEZOLANA RESPECTO A LA OFERTA MONETARIA
}

\author{
Armando Urdaneta Montiel* \\ https://orcid.org/0000-0002-9825-9453 \\ Alberto Castellano Montiel** \\ https://orcid.org/0000-0003-0824-3202 \\ Ronald Prieto-Pulido*** \\ https://orcid.org/0000-0003-3901-4250
}

RECIBIDO: Noviembre 2020 / ACEPTADO: Marzo 2021 / PUBLICADO: Mayo 2021

\begin{abstract}
Como citar: Urdaneta Montiel, Armando; Castellano Montiel, Alberto; Prieto-Pulido, Ronald. (2021). Rezagos de la inflación en la economía venezolana respecto a la oferta monetaria. Telos: revista de Estudios Interdisciplinarios en Ciencias Sociales, 23 (2), Venezuela. (Pp.418-434).

DOI: www.doi.org/10.36390/telos232.13
\end{abstract}

\section{RESUMEN}

La economía venezolana viene presentando una serie de fenómenos que impactan el desenvolvimiento normal de la misma, es por ello que el objetivo del presente artículo fue determinar los rezagos de la inflación en la economía venezolana respecto a la oferta monetaria. Teóricamente se apoya en los postulados de Friedman y Schwartz (1986), Huerta De Soto (2011), Dorta, Guerra \& Sánchez (1997), Levy (2017), entre otros. La investigación es de carácter documental y correlacional. Los resultados evidenciaron que las variaciones trimestrales de la BM sin rezagos explican, el $89 \%$ y $68 \%$ de la varianza de las variaciones trimestrales del INPC, y la cartera de créditos neta; con un grado de asociación o correlación lineal del $94 \%$ y $84 \%$ respectivamente. Lo anterior demuestra que la adopción de una política monetaria en Venezuela de carácter expansivo o restrictivo, de forma inmediata se trasmite al sistema de precios y de créditos de la banca. Se concluye que la visión monetarista continúa teniendo plena vigencia, ya que existen evidencias del nulo efecto de la política monetaria

\footnotetext{
* Postdoctor en Integración y Desarrollo de América Latina, Doctor en Ciencias Económicas, Doctor en Ciencias Gerenciales, Magister en Telemática, Magister en Gerencia Empresarial, Ingeniero en Computación y Profesor Titular de la Universidad Metropolitana del Ecuador y Docente titular invitado en Universidad del Zulia. Correo electrónico: aurdaneta@umet.edu.ec

** Doctor en Ciencias Económicas; Magister en Gerencia de Empresas, mención Gerencia Financiera; Economista; Profesor de Planta Universidad de Sucre (Colombia), Miembro del Grupo de investigación Oikos. Individuos de número de la Academia de Ciencias Económicas del Estado Zulia (miembro de su directiva 2017-2019 y 2019-2021). Correo electrónico: alberto.castellano@unisucre.edu.co

*** Postdoctor en Gerencia de las Organizaciones; Postdoctor en Integración y Desarrollo de América Latina; Doctor en Ciencias Gerenciales; Magister en Gerencia Empresarial; Especialista en Desarrollo Gerencial; Especialista en Gestión Estratégica de Negocios; Economista. Decano Facultad de Administración y Negocios de la Universidad Simón Bolivar, Colombia. Investigador Senior por MinCiencias, adscrito al grupo de investigación Bio-Organizaciones de la Universidad Simón Bolívar, Colombia. Correo electrónico: rprieto1@unisimonbolivar.edu.co
} 
sobre el nivel de actividad económica, incluso en el corto plazo, por la alta correlación y porcentaje de la varianza total explicada entre las variaciones trimestrales de la BM y el INPC.

Palabras clave: Base monetaria, índice de precios al consumidor, cartera de créditos.

\section{Lags of inflation in the Venezuelan economy with respect to the money supply}

\section{ABSTRACT}

The Venezuelan economy has been presenting a series of phenomena that impact its normal development, which is why the objective of this article was to determine the lags of inflation in the Venezuelan economy with respect to the money supply. Theoretically it is based on the postulates of Friedman and Schwartz (1986), Huerta De Soto (2011), Dorta, Guerra \& Sánchez (1997), Levy (2017), among others. The research is documentary and correlational in nature. The results showed that the quarterly variations of the BM without lags explain $89 \%$ and $68 \%$ of the variance of the quarterly variations of the INPC, and the net loan portfolio; with a degree of association or linear correlation of $94 \%$ and $84 \%$ respectively. The foregoing shows that the adoption of an expansive or restrictive monetary policy in Venezuela is immediately transmitted to the price and credit system of the banks. It is concluded that the monetarist vision continues to have total validity, since there is evidence of the null effect of monetary policy on the level of economic activity, even in the short term, due to the high correlation and percentage of the total variance explained between the quarterly variations of the BM and the INPC.

Keywords: Monetary base, consumer price index, credit portfolio.

\section{Introducción}

El presente estudio tiene como objetivo determinar a cuántos rezagos la variación trimestral de la BM se traduce en variaciones trimestrales del INPC y la cartera de créditos neta. También, el estudio busca ver la vigencia de las doctrinas económicas que sirven de sustento en el diseño e implementación de la política monetaria y sus resultados en materia de inflación.

En ese sentido, se han realizado algunas investigaciones previas en la temática como la realizada por Zambrano \& López (2003), en que se identificó una correlación empírica de largo plazo para cada uno de los pares de variables, conformados por las tres mediciones distintas de dinero y las dos de inflación; donde la que la dinámica de corto plazo de la inflación en la economía venezolana se encuentra influenciada, entre otras variables, por dicha relación de largo plazo.

De igual forma Quintero (2015), señala que la actividad económica se contrae debido a un acrecentamiento imprevisto en la tasa de interés como un instrumento de política monetaria de los bancos centrales para frenar la demanda agregada y así evitar problemas de déficit en la balanza comercial, pérdida de activos en reserva, mantener tasas de interés reales positivas y contener la inflación, debido principalmente a la caída del consumo porque encarecer el costo del crédito a corto plazo. Los resultados de la investigación revelan que el canal de transmisión de la política monetaria más relevante es el de la tasa de interés en todos los países sujetos a estudio: Chile, Brasil, Colombia, Perú y México. El estudio observó un 
claro funcionamiento de la primera fase del canal, es decir, la incidencia de la tasa de interés de política monetaria sobre la tasa de interés de los préstamos de los bancos comerciales.

Al respecto de la importancia de la fijación de la tasa de interés por parte de los Bancos Centrales para estimular el ahorro en moneda local, Levy (2017), considera que la gran discrepancia entre el nuevo consenso clásico y la heterodoxia gira en torno a la determinación de la tasa de interés. Los primeros defienden el argumento de la corriente monetaria austro-alemana e indican que la tasa de interés monetaria oscila alrededor de una tasa de interés natural, guiada por la inflación acumulativa y la variación de las reservas de la banca central.

Esta perspectiva fue reemplazada por la Regla de Taylor (1993), la cual sugiere que la tasa de interés nominal ajusta en respuesta a los cambios de la tasa de inflación, y de la diferencia entre el producto observado, el producto potencial y la tasa de interés rezagada. Por su parte, el planteamiento heterodoxo preconiza que la tasa de interés es una variable monetaria, administrada por el banco central, eminentemente distributiva postulando varias reglas para su determinación, donde destaca la Regla de Smithin (2007), que toma en cuenta la demanda/aumento del producto, la distribución del ingreso, tasa de inflación, curva de salario y la función reacción del banco central.

\section{Revisión de la literatura}

Para dar cuenta teórica del escenario de la economía venezolana durante el período 2005-2019, se parte de la presuposición de que la inflación es siempre y en todo lugar un fenómeno monetario, Friedman \& Schwartz (1963). Estos autores demuestran el rol activo de la política Monetaria sobre la actividad económica, indicando que cada ciclo económico que experimentó Estados Unidos encuentra su causa en la inestabilidad de la política monetaria. Esta conclusión para Estados Unidos de América concuerda con la visión austríaca de Mises (1912) y Hayek (1978). Con el primer autor se señala que la expansión de masa monetaria dirigida desde los Bancos Centrales es la principal causante de la volatilidad e inestabilidad de los ciclos económicos, que posteriormente.

Con el segundo autor, se enfatiza la inestabilidad económica causada por el sistema de banca fraccionaria. Las consecuencias monetarias y en la actividad económica de los dos pensadores austriacos antes mencionados, lo hacen extensivo Friedman y Schwartz (1986) en que son los Bancos Centrales los responsables de las crisis hiperinflacionarias, debido de la creación de dinero fiduciario, producto de la intervención del Estado en la política monetaria y su papel de prestamista de última instancia.

Otro autor que aborda las consecuencias negativas de la política monetaria activista en un sistema de banca fraccionaria es Huerta de Soto (2011) quien expone:

El sistema bancario de reserva fraccional genera una oferta extremadamente elástica de dinero, que se ampliaría con facilidad pero que deberá contraerse esforzadamente, produciendo los correspondientes efectos sobre la actividad económica, que estará repetidamente golpeada por sucesivos procesos de expansiones y recesiones. La "maniaca-depresiva" actividad económica con todo su peso de grandes costos sociales, es indudablemente el más severo efecto dañino al sistema bancario existente (basado en reservas fraccionales, 
en violación de principios legales universales de la sociedad)" (Huerta De Soto, 2011: 261).

Friedman (1969), disertó sobre el comportamiento óptimo de la cantidad de dinero para un determinado nivel de precios, en particular. Concluyó que: 1) ambas variables guardan una estrecha relación a largo plazo; 2) los precios en términos de dinero son libres de cambiar, en el sentido de que no hay obstáculos legales para el comercio; 3) hace hincapié en la función de reserva de valor del dinero ante escenarios de expectativas negativas y distinguiendo entre el valor nominal y real de la cantidad de dinero en circulación de una economía.

De esta manera, el argumento fundamental ha sido que los movimientos de los precios reflejan similares cambios en la cantidad de dinero, aunque se presentan cambios por: recortes de divisas: descubrimientos de oro; o cambios en el estándar monetario para la impresión de papel moneda; malas cosechas; interrupciones en el comercio internacional; falta de confianza en el sistemas financiero; las actividades de "especuladores" o "monopolistas"; 0 las actividades de los trabajadores o sindicatos que empujan hacia arriba los sueldos entre otros.

En un trabajo previo, Friedman (1963), relacionaba la inflación con la expansión monetaria relacionada a una política de reducción del desempleo. Esta política de empleo se sustentaba en una política expansiva del gasto público. Lo anterior llevó a Friedman a cuestionar la versión clásica de la curva de Phillips y exponer su visión de expectativas adaptativas y sus efectos inflacionarios.

En dicho contexto, Friedman (1963), recomendaba: 1) establecer límites a los presupuestos públicos, lo cuales deberían evolucionar en términos de crecimiento de manera similar a la renta real de la economía; 2) no financiar déficits presupuestarios mediante la creación de dinero fiduciario; 3) adoptar medidas coercitivas en la economía para contrarrestar los efectos negativos de una política monetaria expansiva, como los controles de precios y salarios, no funcionaban.

En el mismo orden de ideas el autor señala que utilizar la política monetaria como mecanismo para mantener las tasas de interés bajas para incentivar la inversión, termina por destruir todos los incentivos para el ahorro y paradójicamente a una caída de la inversión y el producto real. Es decir, una reducción en la tasa de interés genera una expansión artificial del crédito, financiado con deposito a la vista, mientras que la cartera de crédito producto de la expansión monetaria previa y la expansión artificial del crédito a posterior, en términos reales disminuye a un ritmo acelerado, como ocurrió en el caso venezolano como se puede observar en el gráfico $n^{\circ} 5$.

Por ello, Friedman (1963), señalaba que el control de la base de crédito es una condición indispensable para hacer frente a la inflación, esto se debe a que cuando persiste una condición de inflación, hay una expansión indebida de los gastos, gastos aumentados por una mayor base de crédito.

Estos escenarios similares en la economía de EE.UU. y Reino Unido motivaron a Friedman y Schwartz (1982) a contrastar los postulados del propio Friedman. En ese estudio se retoma el interés sobre la velocidad de circulación del dinero como indicador fundamental que denota el proceso expansivo o contractivo de la actividad económica, por ser la que relaciona el ingreso con la masa monetaria y señala el rezago como un factor determinante, ya 
que un aumento mayor de la masa monetaria respecto al de la producción de bienes y servicios, provocaría un aumento de los precios y por ende una disminución del volumen de transacciones y con ello la velocidad de circulación del dinero se contraería al igual que la actividad económica.

Lo anterior también motivo a los autores antes mencionados a demostrar la correspondencia entre los impulsos monetarios, el aumento general de precios en la economía y en la cartera de créditos. Así, los impulsos monetarios, en el corto plazo, se convierten en fluctuaciones expansivas del nivel del producto, pero en alteraciones de los precios en el largo plazo. El resultado final es el desplome de la demanda de dinero.

\section{Método}

Para el abordaje del presente artículo, es importante precisar los aspectos metodológicos, el cual presenta un enfoque de carácter documental y hermenéutico sobre las variables sujetas a estudio. Los principales antecedentes se hallan a partir del estudio de la literatura preliminar. Así, la hermenéutica fundamentada en el paradigma interpretativo, la cual proviene de la expresión griega hermeneúcin que significa interpretar Ruedas, Ríos y Nieves (2009).

Para Martínez (1999) cuando se trata del acceso al conocimiento, la hermenéutica indica la no existencia de un ser objetivo, transparente y desinteresado sobre el mundo real, motivo por el cual los seres humanos no son espectadores imparciales de los fenómenos sociales, políticos o económicos, entre otros, por ello cualquier conocimiento viene influenciado por un conjunto de prejuicios y expectativas que orientan y limitan nuestra cosmovisión en el arte de comprender la realidad o contexto y buscarle una relación con los conocimientos adquiridos previamente.

Por lo antes descrito, se considera a la hermenéutica una disciplina de la interpretación de los textos, para comprender el enfoque teórico y epistemológico que orientan las ideas centrales y los objetivos centrales que se desarrollan en el texto desde la perspectiva de su autor o autores. De allí que la construcción teórica del discurso represente el elemento esencial donde se develan las dimensiones significantes del estudio a partir de la cosmovisión de los investigadores, sumado a la evidencia empírica que permite confirmar o refutar los preceptos teóricos prestablecidos, Ruedas, Ríos y Nieves (2009).

Esto sin lugar permite generar un producto o construcción de nuevas teorías como resultado de la contratación entre teorías, y de estas con la realidad en aras de desarrollar una explicación con sentido acerca del fenómeno sujeto a estudio en el cual el texto y objeto interpretado, y sujeto interpretante, pertenezcan a un mismo ámbito (Arráez, Calles y Moreno, 2006).

Además de la hermenéutica, la investigación se concibe como estudio correlacional. En ese sentido, se evalúa el comportamiento de la relación directa entre las variaciones de BM, el INPC y la cartera de créditos neta. Se siguió un diseño no experimental (Palella y Martins, 2014), donde no se manipuló las variables involucradas y se analizaron los resultados dentro del contexto donde fueron obtenidos los datos.

En cuanto al procedimiento de investigación, según Hernández et al (2014), los datos fueron proporcionados por fuentes secundarias de información, tomados de los reportes de información estadística suministrados por el Banco Central de Venezuela (en adelante BCV) 
(2020), asociados a la información referente a los agregados monetarios más específicamente la base monetaria (BM) y el índice de precios al consumidor (INPC), por ser el ente rector de la política monetaria en Venezuela, cuya principal función en la economía es propiciar la estabilidad de precios, mediante un control ordenado de la oferta monetaria.

Por otra parte, en lo que respecta a la tercera variable sujeta a estudio, la cartera de créditos, los datos fueron tomados de los reportes de la Superintendencia de las Instituciones del Sector Bancario de Venezuela (SUDEBAN) (2020) ${ }^{1}$ en lo concerniente a los agregados monetarios e indicadores e índices financieros, ya que es ésta quien se encarga de la regulación, supervisión, control y coordinación del Sistema Financiero Nacional.

Cabe destacar, que se revisaron todos los boletines mensuales, en lo que respecta a sus balances generales publicados para los periodos comprendidos entre el 2005 y el 2019 por parte de la Superintendencia de las Instituciones del Sector Bancario de Venezuela (SUDEBAN) (2020) sin excluir ninguno, en lo concerniente a la cuenta de Activos del sector Bancario específicamente la cartera de crédito total.

\section{Resultados}

A continuación, en el gráfico $n^{\circ} 1$ con datos publicados por el BCV (2020) y la Superintendencia de Bancos de Venezuela (2020), en el período 2005-2019, se muestra que la tasa de crecimiento promedio trimestral de BM fue de $64,10 \%$; mientras que la cartera de crédito fue $55,15 \%$. Estas cifras se traducen en un incremento trimestral promedio del nivel general de precios de $74,19 \%$, lo que se comprueba las presuposiciones de la perspectiva monetarista de la escuela de economía de la Universidad de Chicago, y la escuela austriaca.

A lo anterior, luego de revisar trabajos previos sobre estudios de inflación en Venezuela publicados por el mismo BCV tales como el de Dorta, Álvarez \& Bello (2002), en donde existen evidencias que el mayor peso en la explicación de la inflación, descansa en factores de costos como los salarios, el tipo de cambio, y en variables de demanda como el gasto público. Así mismo, Dorta, Guerra \& Sánchez (1997), aseveran que ha sido el deterioro de la credibilidad en la política económica el principal factor que explica la mayor persistencia que muestra la inflación en Venezuela.

\footnotetext{
${ }^{1}$ Producto de la Ley Habilitante promulgada el primero de febrero de 2007 y con vigencia hasta el primero de agosto de 2008, se aprovechó la oportunidad para aprobar la llamada "Ley Orgánica del Sistema Financiero Nacional” (AN, 2010a), promulgada por la Asamblea Nacional con fecha 25 de marzo de 2010, publicada en la Gaceta Oficial de la República Bolivariana de Venezuela $\mathrm{N}^{\circ}$. 39.447 del 16 de junio de 2010. Esa ley tiene como objeto la regulación, supervisión, control y coordinación del Sistema Financiero Nacional, mediante un órgano rector denominado Órgano Superior del Sistema Financiero Nacional (OSFIN), cuyo sub componente en el sector bancario se denomina Superintendencia de las Instituciones del Sector Bancario (AN, 2010b).
} 
Gráfico $\mathrm{n}^{\circ} 1$ : Tasas de crecimiento trimestral promedio de las variables macroeconómicas sujeto a estudio período 2005-2019 y subperíodos 2005-2012 y 2013-2019

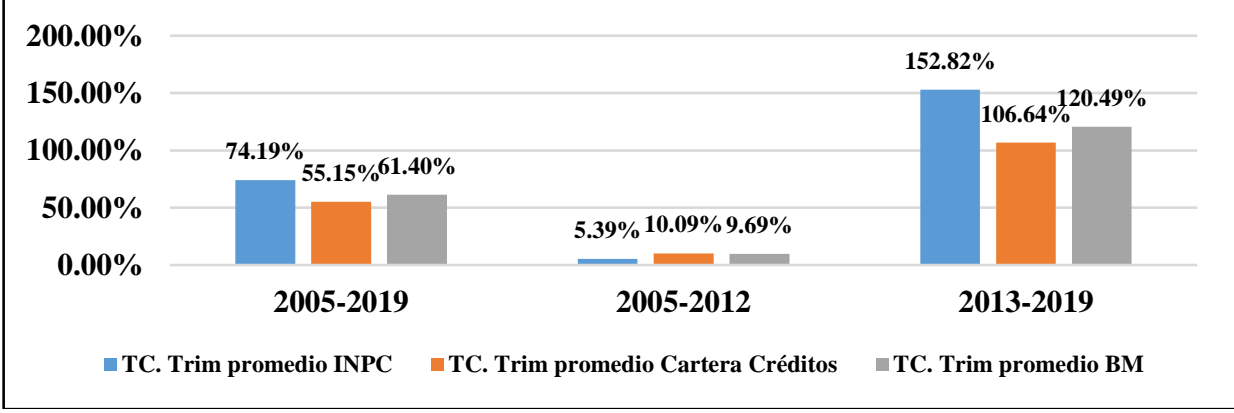

Fuentes: Banco Central de Venezuela (2020) y la Superintendencia de Bancos de Venezuela (2020).

Gráfico n²: Evolución de la demanda de dinero, producto interno bruto real y cartera de crédito real, período 2005-2019.



Fuentes: Banco Central de Venezuela (2020) y la Superintendencia de Bancos de Venezuela (2020).

Al analizar el gráfico $n^{\circ} 2$, se evidencia un proceso de expansión de la economía, medido a través del producto interno bruto real (en adelante PIB) desde el año 2005 hasta el año 2012, producto del aumento internacional de los precios del petróleo y con ello la demanda de dinero, tal como lo señalan Urdaneta \& Borgucci (2018). En esta etapa, la tasa de crecimiento promedio trimestral de la $\mathrm{BM}$ es $9,69 \%$ como se muestra en el gráfico $n^{\circ} 1$; mientras la tasa de crecimiento del INPC y la cartera de crédito nominal fue de 5,39\% y $10,09 \%$ respectivamente, con lo cual el coeficiente de elasticidad de ambas respecto a la BM 
fue 0,6 y 1,9 es decir un aumento del $1 \%$ de la base monetaria se traducía en un incremento del $0,6 \%$ y $1,9 \%$ de la inflación y expansión de la cartera de crédito.

Posteriormente, se da paso al proceso de contracción económica de 2013-2019, como consecuencia de la caída de las exportaciones petroleras, la sobrevaloración del tipo de cambio nominal a partir del tercer trimestre del año 2006. Sin embargo, la política fiscal y monetaria al ser expansivas determinaron una mayor oferta de dinero que, con una mayor demanda de divisas, determinaron por una parte una aceleración de la inflación, devaluación del bolívar y caída significativa de la actividad económica (Urdaneta \& Borgucci (2019).

Esta contracción de la economía agudizo los problemas de déficit fiscales para el sector público, que acentuó la política de monetización del déficit mediante el crecimiento exponencial de la base monetaria con una tasa de crecimiento promedio trimestral de $120,49 \%$. Ello trajo en igual medida un aumento de la tasa de crecimiento promedio trimestral del INPC de $152,82 \%$ y de la cartera de créditos de $106,64 \%$ todo en términos nominales. Por tanto, el coeficiente de elasticidad de ambas respecto a la BM 1,3 y 0,9 , es decir un aumento del $1 \%$ de la BM se traducía en un incremento de $1,3 \%$ y $0,9 \%$ de la inflación y expansión de la cartera de crédito es decir acá ser invertía el proceso, sin ningún rezago de estas dos últimas respecto a la $\mathrm{BM}$; simplemente tan pronto se aumenta la oferta de dinero ello se reflejaba de forma inmediata en la tasa de INPC (ver gráficos $n^{\circ} 3$ y $n^{\circ} 4$ ).

No obstante, en términos reales, como se observa en el gráfico $n^{\circ} 2$, se produce un desplome de la demanda de dinero en moneda doméstica y por ende un mayor aumento de la demanda de divisas y con ello una caída abrupta de la cartera de créditos en términos reales y un aumento recurrente del tipo de cambio nominal. Porque buena parte de las divisas a partir del tercer trimestre del año 2006, estaban destinadas a la compra de divisas para la salida y fuga de capitales (Urdaneta, Borgucci, Moran \& Farinango, 2019).

Todo lo anterior guarda correspondencia con los estudios realizados por Guerra, Olivo \& Sánchez (2003), quienes sugieren que la inflación en Venezuela se explica principalmente por la inercia inflacionaria, el déficit interno y las presiones salariales. Los salarios, que representan una variable de costo, ejercen una influencia sobre la inflación de magnitud ligeramente mayor a la del déficit interno sobre el producto interno bruto. La incidencia del déficit interno como fracción del producto interno bruto es relevante en la explicación de la inflación en Venezuela.

Expresan la importancia de la relación de largo plazo entre inflación y la liquidez monetaria. Es decir, en el largo plazo la inflación es un fenómeno monetario. Los resultados presentados en este trabajo evidencian la necesidad de seguir una línea de investigación sobre políticas de estabilización en un contexto de cierto grado de inercia inflacionaria y presiones de demanda agregada. 
Gráfico $\mathrm{n}^{\circ}$ 3: Evolución de las variaciones trimestrales del INPC respecto al rezago de las variaciones trimestrales de la base monetaria período 2005-2019.
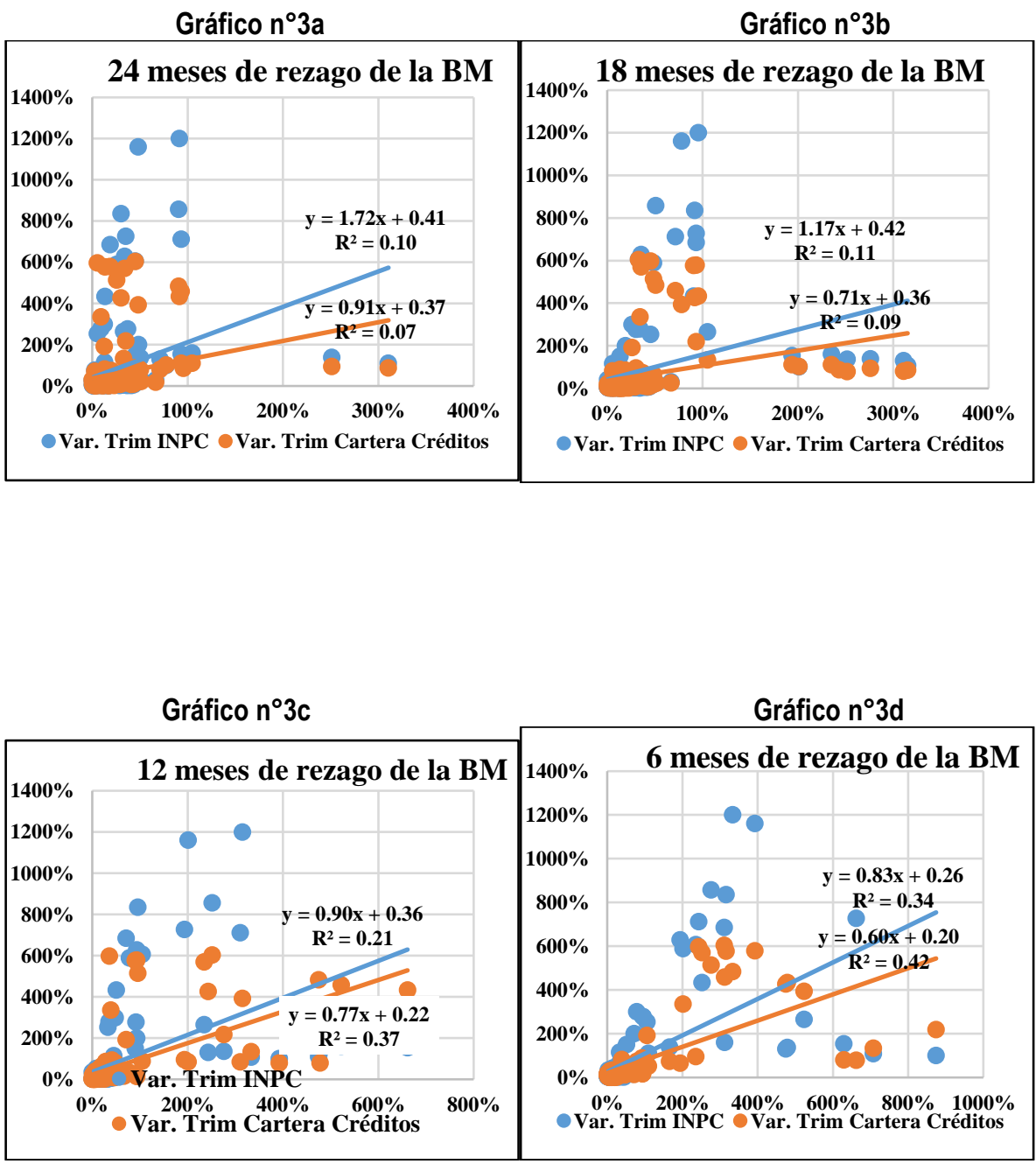

Fuentes: Banco Central de Venezuela (2020)

Al observar el conjunto de subgráficos que conforman el gráfico $n^{\circ} 3$, se puede constatar, partiendo de las premisas de Friedman \& Schwartz (1982), que para el caso venezolano, en la medida que se reduce el rezago de las variaciones trimestrales de la base monetaria respecto a las variaciones trimestrales del INPC y de la cartera de créditos, el coeficiente de determinación $\mathrm{R}^{2}$ se aproxima cada vez más a la unidad; es decir cada vez la 
variaciones trimestrales de la BM explican en mayor porcentaje la varianza de las variaciones trimestrales del INPC y la cartera de crédito.

Siendo la interpretación correcta en el Gráfico $n^{\circ} 3 a$, que las variaciones trimestrales de la BM con un rezago de 24 meses explican levemente el 10\% y $7 \%$ de la varianza de las variaciones trimestrales del INPC y la cartera de créditos, y con un grado de asociación lineal de $32 \%$ y $26 \%$. Respectivamente. El Gráfico $n^{\circ} 3 b$, muestra que las variaciones trimestrales de la BM con un rezago de 18 meses revelan escasamente $11 \%$ de la varianza de las variaciones trimestrales del INPC y $9 \%$ de la cartera de créditos y con un grado de asociación lineal de $33 \%$ y $30 \%$ comparativamente como se evidencia en la tabla $n^{\circ} 1$.

Así mismo, el Gráfico $n^{\circ} 3 c$, las variaciones trimestrales de la BM con un rezago de 12 meses expresan limitadamente $21 \%$ de la varianza de las variaciones trimestrales del INPC y $37 \%$ de la cartera de crédito, y con grado de asociación lineal de $46 \%$ y $61 \%$ respectivamente como se muestra en la tabla $n^{\circ} 1$. Por su parte, el Gráfico $n^{\circ} 3 d$, muestra que las variaciones trimestrales de la BM con un rezago de 6 meses explican moderadamente 34\% de la varianza de las variaciones trimestrales de INPC y $42 \%$ para la cartera de crédito, y con grado de asociación o correlación lineal de $58 \%$ y $65 \%$ respectivamente.

Finalmente, en el gráfico, $n^{\circ} 4$ se evidencia que las variaciones trimestrales de la BM sin rezagos explican $89 \%$ y $68 \%$ de la varianza de las variaciones trimestrales del INPC, y la cartera de créditos neta, con un grado de correlación lineal de $94 \%$ y $82 \%$ correspondientemente como se observa en la tabla $n^{\circ} 1$. Lo anterior demuestra que la economía venezolana tiene como característica que una vez que el Banco Central de Venezuela adopta una política monetaria expansiva o restrictiva, de forma inmediata se trasmite al sistema de precios y de la cartera de créditos de la banca en la economía.

Gráfico $\mathrm{n}^{\circ} 4$ : Evolución de las variaciones trimestrales del INPC y Cartera de créditos sin rezago de las variaciones trimestrales de la base monetaria período 2005-2019.

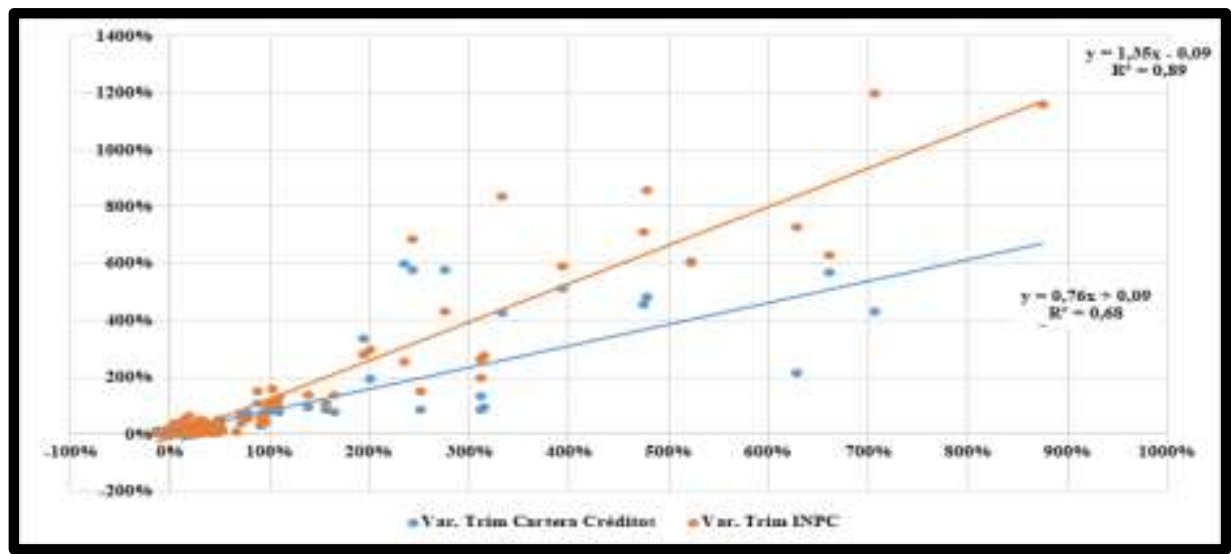

Fuentes: Banco Central de Venezuela (2020).

Tabla $\mathrm{n}^{\circ} 1$ : Correlaciones entre las variables sujetas a estudio 
Rezagos de la inflación en la economía venezolana respecto a la oferta monetaria

\begin{tabular}{|c|c|c|c|}
\hline \multicolumn{4}{|c|}{ Correlaciones } \\
\hline & & $\begin{array}{l}\text { Var. Trim } \\
\text { INPC }\end{array}$ & $\begin{array}{l}\text { Var. Trim Cartera } \\
\text { Créditos }\end{array}$ \\
\hline Var. Trim BM 24 Rezagos & Correlación de Pearson & 0,32 & 0,26 \\
\hline Var. Trim BM 18 Rezagos & Correlación de Pearson & 0,33 & 0,30 \\
\hline Var. Trim BM 12 Rezagos & Correlación de Pearson & 0,46 & 0,61 \\
\hline Var. Trim BM 6 Rezagos & Correlación de Pearson & 0,58 & 0,65 \\
\hline \multirow[t]{2}{*}{ Var. Trim BM Sin Rezagos } & Correlación de Pearson & 0,94 & 0,82 \\
\hline & $\mathrm{N}$ & 180 & 180 \\
\hline
\end{tabular}

Fuente: elaboración propia en SPSS.

De allí, lo expuesto por Urdaneta, Castellano \& Prieto (2015), donde se utilizaron los agregados monetarios del BCV y se analizaron las cien últimas variaciones trimestrales del INPC, el tipo de cambio implícito y la correlación existente entre ellas; se encontró que existe una variación muy similar entre ambas variables estudiadas, con valores promedio inter trimestral de $7,87 \%$ para la tasa del INPC y de $8,69 \%$ para la tasa de cambio implícito, de acuerdo con el nivel de BM, dividido entre el nivel de reservas internacionales con un grado de correlación lineal entre ellas de 0,93.

Esa política monetaria de carácter expansivo, donde la BM crece más rápido que incluso la cartera de crédito en términos nominales, sumado a los altos índices de inflación termina por generar problemas adicionales no menores como la escasez de efectivo (Urdaneta, Borgucci \& Mejía, 2020). Es decir, se muestra evidencias de que la tasa de variación promedio inter-trimestral de la BM es superior al de la liquidez monetaria (en adelante M2) y el circulante (en adelante M1), produciéndose una caída del efectivo disponible en poder de la banca, las monedas y billetes en circulación entre un $40 \%$ y $30 \%$ respecto al tamaño de la BM.

Gráfica 5: Agregados financieros a precios constantes año base 2007, período 2005-2019

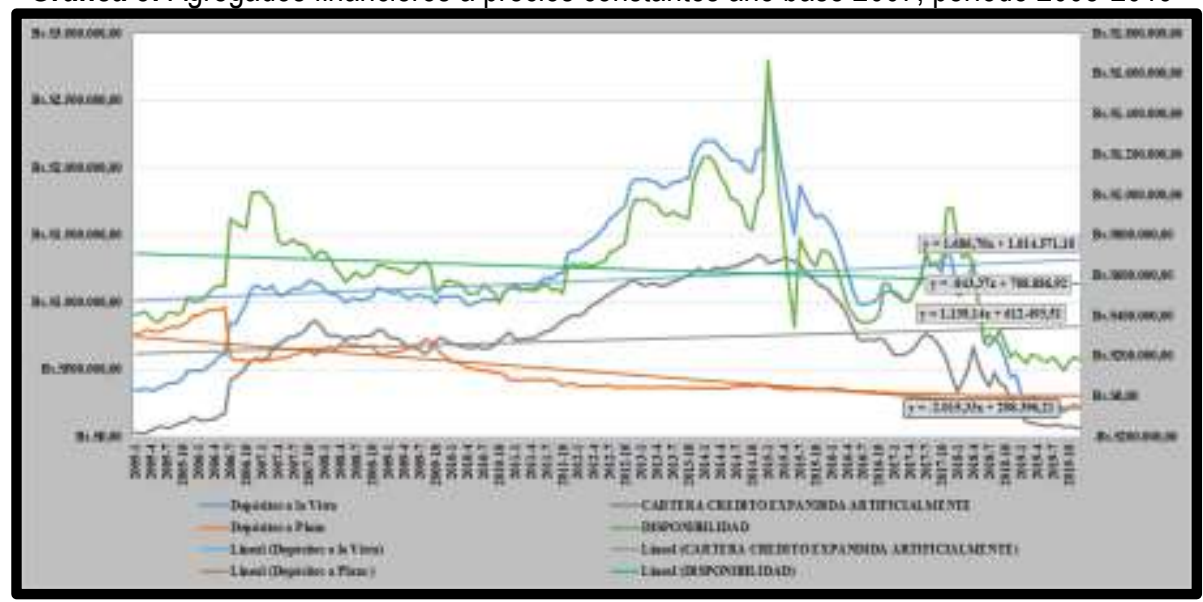

Fuente: Sudeban. Superintendencia de Bancos (2017) 
En la gráfica 5 se puede evidenciar el progreso de los agregados financieros medidos en términos reales, tomando como año base 2007. En el caso de los depósitos a la vista (en adelante DV), la cartera de créditos neta expandida artificialmente (en adelante CCNEA) y la disponibilidad financiera (en adelante DF), las series de tiempo muestran un componente tendencial ascendente y cíclico porque oscilan por encima y por debajo de la línea de tendencia en períodos superiores a un año.

En el caso de los DV, presentan un ciclo expansivo que comienza en el trimestre 2011-4 y termina en el 2016-1. En dicho período, en términos reales, los mismos crecieron por encima de su línea de tendencia para el lapso 2005-2019. De igual forma la CCNEA muestra un crecimiento similar para el lustro 2011-3 al 2016-3, la misma se incrementó por arriba de su de su línea de tendencia para el lustro 2005-2019. En ese mismo orden ideas, la DF, comenzó a crecer por encima de su valor promedio a partir del trimestre 2011-4 hasta el 2019-4, que es cuando supera a la CCNEA, producto de la contracción del crédito en términos reales, debido a la recesión económica existente (Urdaneta, Prieto, \& Hernández, 2017).

No obstante, mientras todos los agregados analizados en términos reales presentan un componente tendencial ascendente, los depósitos a plazo (en adelante DP), muestran un componente tendencial descendente con pendiente negativa y cíclica porque oscilan por encima y por debajo de la línea de tendencia en períodos superiores a un año. Sin embargo, los mismos a partir del trimestre 2010-3 comienzan una abrupta caída por debajo de su de su línea de tendencia para el período 2005-2019, llegando a ser pocos representativos para el análisis, más allá de que su desplome evidencie una mayor expansión artificial del crédito el cual, al cierre de 2017-4, se financiaba con $99,69 \%$ de DV, mientras que en 2005-1 con $41,37 \%$.

Así mismo Urdaneta (2017) afirma que:

"La política monetaria expansiva instrumentada en el período 1984-2013 por el ente rector de la misma, a través de los canales de transmisión de la tasa de interés y tipo de cambio, no se tradujeron en estabilidad precios y preservación de la función de reserva de valor del signo monetario nacional; sino por el contrario, el aumento de la oferta monetaria en más de un $687.041,51 \%$, generó tasas de interés reales negativas, devaluación de la tasa de cambio implícita en $436.828,72 \%$, provocando procesos recurrentes de estanflación con una inflación acumulada de $286.844,06 \%$, con apenas un crecimiento del Producto Interno Bruto Real (PIBR) del $168,99 \%$ y un aumento de la demanda agregada interna del $218,44 \%$, muy por encima del PIBR, con un crecimiento poco significativo de las exportaciones netas" (Urdaneta, 2017: 93).

Ahora bien, la pregunta que cabría hacerse desde el punto de vista de la racionalidad económica es ¿Por qué la política monetaria del BCV desde su estatización en 1974 ha sido recurrentemente de carácter expansivo? La explicación pudiera encontrarse en el planteamiento desarrollado por Milei (2018), específicamente en el punto donde hace referente al debate sobre la inflación. En ese debate, el cual varios economistas pretendían ofrecer soluciones al gobierno del presidente Richard Nixon para controlar los altos índices de inflación recurrente en la década de los 70 's. El primeo de ellos James Tobin, proponía la 
implementación de política de ingreso para controlar la inflación; ya que, según él, la principal fuente de variación de los precios obedecía a la presión de los costos.

Posteriormente, Paul Samuelson reseñaba que este proceso inflacionario obedecía a las presiones de los gremios sobre los aumentos salariales, por lo que se requería una política de ingreso exitosa, con controles de precio cada vez menos tolerantes hacia grupos industriales para frenar las acciones de fijación de precios por parte de monopolios y oligopolios, incluso recomendó experimentar con recompensas impositivas, a quienes actuaran con moderación en el establecimiento de precios y salarios (Milei, 2018). En la misma corriente, se uniría Arthur Okun quien avaló que la administración de Richard Nixon impusiera el congelamiento de precios y salarios como medida anti-inflacionaria, continuando en la línea de apoyo a las políticas de ingreso que sirven de guías diseñadas a la evolución de salarios y compatibilidad de incentivos en lugar de utilizar como herramienta los controles de precio, Okun era un gran crítico del uso de políticas monetarias para controlar la inflación (Milei, 2018).

A esta camada de economistas, se uniría Walter Heller asesor económico presidencial del Gobierno de John F. Kennedy, quien planteaba a la administración de Richard Nixon, que el congreso debía tener en cuenta que un programa económico que buscase las condiciones de pleno empleo con niveles de inflación moderados debía contener restricciones en materia de aumentos de precios derivado de la concentración industrial y aumentos salariales exigidos por los poderosos sindicatos (Milei, 2018).

Finalmente, Gardner Ackley complementaría a este grupo de economistas quien fuera asesor económico presidencial del Gobierno de Lyndon B. Johnson. Ackley afirmaba que las decisiones de política económica dependían en parte de la proposición según la cual la economía, se encuentra en estado de exceso de demanda. De esta manera, la política recomendada era: 1) en una primera etapa, el congelamiento de los precios; 2) en una segunda etapa, reemplazar la medida anterior por una política de congelamiento de ingresos Milei (2018). El resultado de tales medidas y recomendaciones es que la economía de EEUU padeciera los índices de inflación más altos en los últimos 60 años como se muestra en el gráfico $n^{\circ} 6$.

Gráfica 6: Tasa de inflación de la economía norteamericana 1956-2019

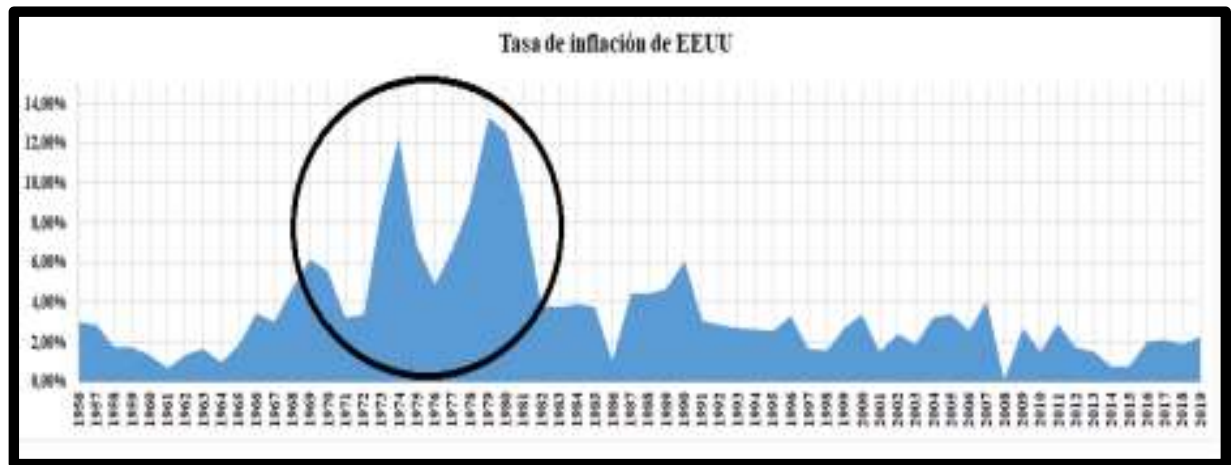

Fuente: https://es.inflation.eu/tasas-de-inflacion/estados-unidos/inflacion-historica/ipc-inflacionestados-unidos.aspx 
En contraposición a esta heterodoxia Keynesiana cuya evidencia empírica prueba serias dificultades para controlar la inflación, surgiría el enfoque monetarista de Milton Friedman (1969), quien rechazará los controles de precio y salarios para dominar la inflación. Las razones de la argumentación de Friedman son: 1) los controles atentaban contra las señales que brinda el sistema de precios; 2) crean desabastecimiento; 3) atentan contra la calidad de los bienes; 4) se genera incentivos para que los políticos se vean tentados a expandir aún más la demanda agregada, mediante una mayor emisión monetaria.

En ese sentido, Rallo (2011), señala lo siguiente:

Los errores de la teoría de precios de Keynes pueden resumirse prácticamente en dos, por un lado razona en términos de niveles generales de precios y de unidades agregadas de costos, cuando lo relevante para los empresarios son los precios y costos concretos y relativos de las diferentes industrias ( en particular, los variados márgenes entre los precios y los costos); por otro, la relación correcta entre precios y costos no es la de que los costos determinen los precios, sino al revés: son los precios los que en última instancia determinan los costos (Rallo, 208).

Lo anterior se conoce como la ley de imputación de Menger; Mises (1980), destaca que:

A Menger \& Bohm-Bawerk. Su principal mérito consiste en señalar que la determinación de los precios está inextricablemente ligada a la propia dinámica del mercado. Distinguiendo claramente entre: a) la valoración directa de los factores de producción que relaciona el valor del correspondiente producto con el conjunto de los utilizados factores complementarios de producción, y b) el precio de los diversos factores de producción, que queda en el mercado determinado por las postulaciones de quienes por ellos compiten (Mises, 1980: 505).

Al respecto, Rallo (2011), señala que Keynes con su teoría de los precios y los salarios, pretendió ligar el nivel general de precios con la renta agregada. En condiciones de equilibrio con desempleo involuntario, los incrementos en la cantidad de dinero no generarían en inflación, sino aumentos de la producción, y la reducción en los costos no darían lugar a una mayor ocupación sino caídas en la demanda agregada y de los precios (deflación) que dejarían los salarios reales inalterados 0 incluso los incrementarían. Esta idea, anula el principal mecanismo de ajuste que existe en las economías capitalistas para coordinar de manera consciente los planes de todos los individuos. Es precisamente, la flexibilidad de precios y costos las que permiten emplear todos los factores productivos que sean capaces de generar más riqueza de la que demandan en forma de remuneración.

\section{Conclusión}

Se puede concluir entonces que en el período sujeto a estudio 2005-2019, ha existido un grado de asociación directa sin ningún tipo de rezago inflacionario entre el crecimiento promedio trimestral de la BM de $64,10 \%$, la cartera de crédito con $55,15 \%$; y el 
INPC de 74,19\%; existiendo evidencia importante de que los preceptos que fundamentan las teorías monetaristas de la escuela de economía de la universidad de Chicago, y la escuela austriaca explican dicha relación.

Se pudo evidenciar que se le confiere un papel poco relevante a la política monetaria expansiva como un factor causal de la inflación. Solo se le confiere una relación a largo plazo con la liquidez monetaria. Sin embargo, los estudios concuerdan que son los costos, los salarios, gasto público y déficit público como fracción del producto interno bruto, las principales causas de la inflación en la economía venezolana en el corto y mediano plazo. Esto guarda total semejanza con la heterodoxia keynesiana y su teoría de precios, razón por la cual incluso en períodos de plena expansión económica el país en los últimos 40 años presenta índices de inflación similares a la economía de Estados Unidos de América en la década de los 70's.

Esto ha ocurrido porque la doctrina económica que ha prevalecido en el BCV se ha sustentado en la tradición keynesiana de autores como James Tobin, Paul Samuelson, Arthur Okun, Walter Heller \& Gardner Ackley; que se ha contrastado fuertemente con la perspectiva monetarista de Milton Friedman.

En el caso que compete al presente estudio, la heterodoxia keynesiana, en aquel momento pareciera que no tenía la respuesta adecuada al fenómeno de la inflación, por el limitado efecto de la política monetaria sobre el nivel de actividad económica incluso en el corto plazo, debido a la alta correlación y porcentaje de la varianza total explicada entre las variaciones trimestrales de la base monetaria, el INPC y la cartera de créditos.

\section{Referencias bibliográficas}

Arráez, Morella; Calles, Josefina y Moreno de Tovar, Liuval. (2006). La Hermenéutica: una actividad interpretativa. SAPIENS, 7(2), Venezuela. (Pp.171-181). Disponible en: http://ve.scielo.org/scielo.php?script=sci arttext\&pid=S1317-58152006000200012

BCV (Banco Central de Venezuela) (2020). Indicadores económicos. Venezuela. Disponible en: http: //www.bcv.org.ve/c2/indicadores.asp.

Dorta, Miguel; Álvarez, Fernando y Bello, Omar. (2002). Determinantes de la inflación en Venezuela: un análisis macroeconómico para el período 1986-2000. (Edición $n^{\circ} 37$ ). Serie Documentos de Trabajo Oficina de Investigaciones Económicas. Venezuela.

Dorta Miguel; Guerra José y Sánchez Gustavo. (1997). Credibilidad y persistencia de la inflación en Venezuela. (Edición $n^{\circ} 11$ ). Serie Documentos de Trabajo Oficina de Investigaciones Económicas. Venezuela.

Friedman, Milton. (1963). Inflation: Causes and Consequences. Asia Publishing House for the Council for Economic Education. India.

Friedman, Milton. (1969). The Optimum Quantity of Money and Other Essays. (Seven edition). Aldine Publishing Company. Estados Unidos.

Friedman, Milton., \& Schwartz, Anna. (1986). Has government any role in money. Journal of Monetary Economics, 17(1). Estados Unidos. (Pp.37-62). https://doi.org/10.1016/0304-3932(86)90005-X

Friedman, Milton; \& Schwartz, Anna. (1963). A Monetary History of the United States 18671970. (First edition). Princeton University Press. Estados Unidos. 
Friedman, Milton; \& Schwartz, Anna. (1982). Monetary Trends in the United States and the United Kingdom: Their Relation to Income, Prices and Interest Rates 1867-1975. (First edition). University of Chicago Press. Estados Unidos.

Guerra José; Olivo Víctor \& Sánchez Gustavo. (2003). El proceso inflacionario en Venezuela: un estudio con vectores autorregresivos. Guerra José. (Compilador). Estudios sobre la inflación en Venezuela. Colección económico-financiera del BCV. Venezuela.

Hayek, Friedrich (1978). Denationalisation of Money Then Argument Refined: An Analisys of Theory. (Third edition) The Institute of Economic Affairs. Reino Unido.

Hernández, Roberto; Fernández, Carlos y Baptista, Pilar. (2014). Metodología de la investigación. (Quinta edición), Editorial McGraw-Hill. México.

Huerta De Soto, Jesús. (2011). Dinero, crédito bancario y ciclos económicos. (5ta edición). Unión Editorial. España.

Levy, Noemi. (2017). Política monetaria: qué relación tiene con el crecimiento y la estabilidad.

Revista Economía Informa. Volumen 404. México. (Pp. 18-24).

Martínez, Miguel. (2006). La nueva ciencia. Su desafío, lógica y método. (Primera edición). Trillas-México.

Milei, Javier. (2018). Desenmascarando la mentira keynesiana. (Edición $n^{\circ} 33$ ). Unión Editorial. España.

Mises Ludwid. (1980). La Acción Humana. (Cuarta Edición). Unión Editorial. España.

Mises, Ludwid (1912). Theorie des Geldes und der Umlaufsmittel. (First edition). Ludwig von Mises Institute. Estados Unidos.

Palella, Santa \& Martins, Filiberto. (2012). Metodología de la investigación cuantitativa. (Quinta edición). Editorial FEDUPEL. Venezuela.

Quintero, Jorge. (2015) Impactos de la política monetaria y canales de transmisión en países de América Latina con esquema de inflación objetivo. Revista Ensayos sobre
Política
Económica, 33(76).
Colombia.
(Pp.61-75).

https://doi.org/10.1016/i.espe.2015.02.001

Rallo, Juan. (2011). Los errores de la vieja economía. (Segunda Edición). Unión Editorial. España.

Ruedas, Martha; Ríos, María y Freddy Nieves (2009). Hermenéutica: la roca que rompe el espejo. Revista Investigación y Postgrado, 24(2). Venezuela (Pp. 181-201). Disponible en: $\quad$ http://ve.scielo.org/scielo.php?pid=S131600872009000200009\&script=sci_arttext\&tlng=en

Smithin, John. (2007). A real interest rate rule for monetary policy. Journal of Post Keynesian Economics, 30(1). Estados Unidos. (Pp.101- 118). Disponible en: https://www.tandfonline.com/doi/abs/10.2753/PKE0160-3477300105

Sudeban-Superintendencia de Bancos y otras instituciones de crédito. (2020). Cartera de créditos. Disponible http://www.sudeban.gob.ve/index.php/gide_informacionestadistica/.

Taylor, John. (1993, December). Discretion versus policy rules in practice. In CarnegieRochester conference series on public policy. Volumen 39. Holland. (Pp. 195214). https://doi.org/10.1016/0167-2231(93)90009-L 
Urdaneta, Armando. (2017). Mecanismos de transmisión de la política monetaria en la economía venezolana 1984-2013. Revista Innovación y Gerencia, 9(1), Venezuela. (Pp. 69-95).

Urdaneta, Armando y Borgucci, Enmanuel. (2018). Shocks petroleros y demanda de dinero en Venezuela 1988-2017. Revista de Ciencias Sociales, 24(3). Venezuela. (Pp.108126). Disponible en: https://www.redalyc.org/jatsRepo/280/28059580011/html/index.html.

Urdaneta, Armando y Borgucci, Enmanuel. (2019). Determinantes de la iliquidez de divisas en Venezuela: período 1988-2017. Revista Venezolana de Gerencia, 24(86). Venezuela.

(Pp.523-546). Disponible en: https://produccioncientificaluz.org/index.php/rvg/article/view/23778.

Urdaneta, Armando; Borgucci, Enmanuel y Mejía, Omar. (2020). La Inflación y disponibilidad de efectivo en la economía venezolana. Ciencia UNEMI. 13(32). Ecuador. (Pp. 5162). Disponible en: http://ojs.unemi.edu.ec/index.php/cienciaunemi/article/view/997

Urdaneta, Armando; Castellano, Alberto y Prieto, Ronald. (2016). Relación entre el tipo de cambio implícito y el índice de precios al consumidor en Venezuela. Período 19892014. Revista Desarrollo Gerencial, 8(1). Colombia. (Pp. 49-70). https://doi.org/10.17081/dege.8.1.1406

Urdaneta, Armando; Borgucci, Enmanuel; Moran, Gracian y Farinango, Ronny. (2019). Dolarización de la economía venezolana desde el enfoque de la demanda de dinero. Revista de Ciencias Sociales, 25(1). Venezuela. (Pp.114129). https://doi.org/10.31876/rcs.v25i1.29602

Urdaneta, Armando; Prieto, Ronald y Hernández, Orlando. (2017). Formación bruta de capital fijo en el producto interno bruto venezolano en el período 1997-2015. Revista Desarrollo Gerencial, 9(1). Colombia. (Pp. 52-80). https://doi.org/10.17081/dege.9.1.2725

Verón, Eliseo. (1998). La Semiosis social: fragmentos de una teoría de la discursividad. Editorial Gedisa. España.

Zambrano Omar; López Oswaldo. (2003) Relación de corto y largo plazo entre agregados monetarios e inflación en Venezuela: algunas consideraciones empíricas. (Edición $n^{\circ} 49$ ) Serie Documentos de Trabajo Oficina de Investigaciones Económicas. Venezuela. 